\title{
GAIA Insufficient Evidence for Diagnosis of Gestational Hypertension
}

National Cancer Institute

\section{Source}

National Cancer Institute. GAIA Insufficient Evidence for Diagnosis of Gestational

Hypertension. NCI Thesaurus. Code C128011.

GAIA Insufficient Evidence for Diagnosis of Gestational Hypertension is defined by two criteria: first, a pregnancy with gestational age greater than or equal to 20 weeks; second, one or more of the following requirements: a) The inability to measure blood pressure; b) The inability to evaluate for proteinuria. 